\title{
Vasorelaxing Effect of Hypoxia via Rho-kinase Inhibition on the Agonist-specific Vasoconstriction
}

\author{
Hyun Dong JE* and Chang Yell SHIN \\ *Department of Pharmacology, College of Pharmacy, Catholic University of Daegu, Gyeongbuk, 712-702, \\ Korea and Research Laboratory, Dong-A Pharm. Co. Ltd. Kyunggido, 446-905, Korea
}

(Received August 12, 2008; Revised August 13, 2008; Accepted August 27, 2008)

\begin{abstract}
The present study was undertaken to determine whether hypoxia influences on the agonist-induced vascular smooth muscle contraction and, if so, to investigate the related mechanism. The measurement of isometric contractions using a computerized data acquisition system was combined with molecular experiments. Hypoxia significantly inhibited fluoride-induced contraction regardless of endothelial function, but there was no relaxation on thromboxane $\mathrm{A}_{2}$ mimetic U-46619-induced contraction suggesting that other pathway such as $\mathrm{Ca}^{2+}$ entry or thin filament regulation was not affected. In addition, hypoxia significantly decreased fluorideinduced increase of phospho-myosin-targeting subunit of myosin light chain phosphatase (pMYPT1). Interestingly, hypoxia didn't inhibit significantly phenylephrine-induced contraction suggesting that myosin light chain kinase (MLCK) activity or thin filament regulation is less important on the hypoxia-induced vasorelaxation in the denuded muscle than Rho-kinase activity. In conclusion, this study provides the evidence and possible related mechanism concerning the vasodilation effect of hypoxia on the agonist-specific contraction in rat aortic rings regardless of endothelial function.
\end{abstract}

Keywords: Fluoride, Hypoxia, Rho-kinase, Thromboxane $\mathrm{A}_{2}$ mimetic, Vasodilation

\section{INTRODUCTION}

Systemic vessels such as arteries or arterioles lie within the tissue they are supplying and can be acted on by local factors within the tissue relax in response to acute hypoxia (Shimizu, et al., 2000), which leads to a better tissue perfusion (Young and Vatner, 1986). It was reported that there was no decrease in intracellular $\mathrm{Ca}^{2+}$ in hypoxia-induced vascular relaxation (Ishida and Honda, 1992; Aalkjaer and Lombard, 1995; Shimizu, et al., 2000). Since $\mathrm{Ca}^{2+}$ remains elevated and force decreases, it can be called as $\mathrm{Ca}^{2+}$ desensitization (Shimizu, et al., 2000).

It is generally accepted that the initiation of smooth muscle contractility is predominantly controlled by a $\mathrm{Ca}^{2+}$ dependent increase in myosin light chain $20 \mathrm{kDa}\left(\mathrm{MLC}_{20}\right)$ phosphorylation (Somlyo and Somlyo, 1994). However, other pathways have now been described that may regulate the contractility of smooth muscle by regulating the

\footnotetext{
${ }^{*}$ Corresponding author

Tel: +82-53-850-3615, sFax: +82-53-850-3602

E-mail: hyundong@cu.ac.kr
}

phosphorylation of $\mathrm{MLC}_{20}$ independently of a rise in intracellular $\mathrm{Ca}^{2+}$ (Somlyo and Somlyo, 1998; Uehata, et al., 1997; Sakurada, et al., 2003). The phosphorylation of $\mathrm{MLC}_{20}$ promotes the interaction of actin and myosin II, and the contraction of smooth muscle. The degree of $\mathrm{MLC}_{20}$ phosphorylation or contraction does not always parallel the $\mathrm{Ca}^{2+}$ concentration. The extent of $\mathrm{MLC}_{20}$ phosphorylation or force of contraction induced by agonist stimulation is usually higher than that caused by an increase in the $\mathrm{Ca}^{2+}$ concentration, a finding explained by so-called $\mathrm{Ca}^{2+}$ sensitization (Somlyo and Somlyo, 1994). Thus, an additional mechanism of regulation that modulates the levels of $\mathrm{MLC}_{20}$ phosphorylation and degree of contraction has been proposed. Subsequent studies have revealed that inhibition of MLC phosphatase by Rhokinase (Kitazawa, et al., 1991) or thin filament regulation including activation of protein kinase $\mathrm{C}$ (PKC), mitogenactivated protein kinase kinases (MEK) and extracellular signal regulated kinase (ERK) 1/2, and phosphorylation of the actin binding protein caldesmon (Wier and Morgan, 2003) may be the major pathway in $\mathrm{Ca}^{2+}$ sensitization.

In various smooth muscles, phenylephrine has been shown to induce contraction, which may be due to 
enhancement of $\mathrm{Ca}^{2+}$ sensitivity. Phenylephrine has been known to induce contraction in blood vessel preparations leading to either thick or thin filament regulation. Thick or myosin filament regulation encompasses both $\mathrm{Ca}^{2+}$ activation and $\mathrm{Ca}^{2+}$ sensitization as it involves both activation of MLCK by $\mathrm{Ca}^{2+}$-calmodulin and regulation of MLCP activity. On the other hand, thin or actin filament regulation includes the possible disinhibition of actin-myosin interactions by phosphorylation of caldesmon, possibly by PKC, MEK or ERK that are translocated during their activation (Gerthoffer, et al., 1996). It is possible that fluoride- or thromboxane $A_{2}$ mimetic-induced contractions involve the participation of the RhoA/Rho-kinase pathway (Tsai and Jiang, 2006; Jeon, et al., 2006). Therefore, the aim of the present study was to elucidate the possible role of thick filament regulation such as Rhokinase inhibition or thin filament regulation on $\mathrm{Ca}^{2+}$ desensitization in hypoxia-induced relaxation of isolated rat aortae using RhoA/Rho-kinase activators such as fluoride or thromboxane $A_{2}$ mimetic.

\section{Materials and methods}

\section{Tissue preparation}

Male Sprague-Dawley rats, weighing 320-350 g, were anesthetized by sodium pentobarbital (50 mg/kg i.p.) followed by cervical dislocation, in agreement with procedures approved by the Institutional Animal Care and Use Committee. The thoracic aorta was quickly removed and immersed in oxygenated $\left(95 \% \mathrm{O}_{2} / 5 \% \mathrm{CO}_{2}\right)$ physiological saline solution composed of $(\mathrm{mM}): 115.0 \mathrm{NaCl}, 4.7$ $\mathrm{KCl}, 2.5 \mathrm{CaCl}_{2}, 1.2 \mathrm{MgCl}_{2}, 25.0 \mathrm{NaHCO}_{3}, 1.2 \mathrm{KH}_{2} \mathrm{PO}_{4}$, and 10.0 dextrose (pH 7.4). The aorta was cleaned of all adherent connective tissue, and the endothelium was removed by gentle abrasion with a cell scraper.

\section{Contraction measurements}

Sometimes care was taken to avoid rubbing the endothelial surface of the vessels that had intact endothelium. Two stainless-steel triangles were inserted through each vessel ring. Each aortic ring was suspended in a waterjacketed organ bath $(20 \mathrm{ml})$ maintained at $37^{\circ} \mathrm{C}$ and aerated with a mixture of $95 \% \mathrm{O}_{2}$ and $5 \% \mathrm{CO}_{2}$. One triangle was anchored to a stationary support, and the other was connected to an isometric force transducer (Grass FT03C, Quincy, Mass., USA). The rings were stretched passively by imposing the optimal resting tension, $2.0 \mathrm{~g}$, which was maintained throughout the experiment. Each ring was equilibrated in the organ bath solution for $60 \mathrm{~min}$ before the experiment involving the contractile response to $50 \mathrm{mM}$
$\mathrm{KCl}$ addition. Isometric contractions were recorded using a computerized data acquisition system (PowerLab/8SP, ADInstruments, Castle Hill, NSW, Australia).

After completion of the control $\mathrm{KCl}$ dose-response curve, vessel rings were pre-incubated with hypoxia or no aeration. Aeration or no aeration was applied $1 \mathrm{~min}$ before the addition of fluoride, thromboxane $\mathrm{A}_{2}$ mimetic or phenylephrine. The absence of the endothelium was verified by the lack of relaxation after the addition of acetylcholine $(1 \mu \mathrm{M})$ to precontracted ring segments.

\section{Western blot analysis}

Muscle strips were quick frozen by immersion in a dry ice/acetone slurry containing $10 \%$ trichloroacetic acid (TCA) and $10 \mathrm{mM}$ dithiothreitol (DTT). Muscles were stored at $-80^{\circ} \mathrm{C}$ until used. Tissues were brought to room temperature in a dry ice/acetone/ TCA/DTT mixture. Then samples were homogenized in a buffer containing $20 \mathrm{mM}$ mops, 4\% SDS, 10\% glycerol, 10 mM DTT, $20 \mathrm{mM} \beta$-glycerophosphate, $5.5 \mu \mathrm{M}$ leupeptin, $5.5 \mu \mathrm{M}$ pepstatin, $20 \mathrm{KIU}$ aprotinin, $2 \mathrm{mM} \mathrm{Na}_{3} \mathrm{VO}_{4}, 1 \mathrm{mM} \mathrm{NaF}, 100 \mu \mathrm{M} \mathrm{ZnCl}, 20$ $\mu \mathrm{M}$ 4-(2-aminoethyl) benzenesulphonyl fluoride (AEBSF) and $5 \mathrm{mM}$ EGTA. Protein-matched samples (modified Lowry protein assay, DC Protein Assay Kit, Bio-Rad) were electrophoresed on SDS-PAGE (Protogel, National Diagnostics), transferred to PVDF membranes and subjected to immunostaining and densitometry, as above, using the appropriate antibody. The success of protein matching was confirmed by Naphthol Blue Black staining of the membrane and densitometry of the actin band. Any mismatch of lane loading was corrected by normalization to actin staining. Each set of samples from an individual experiment was run on the same gel and densitometry was performed on the same film.

\section{Chemicals and antibodies}

The drugs and chemicals were reagent grade unless otherwise indicated and obtained from the following source. Sodium fluoride, U-46619, $\mathrm{KCl}$, acetylcholine, Y27632 and nicardipine were purchased from Sigma Co. (St. Louis, MO, USA). DTT, TCA and acetone were obtained from Fisher Scientific (Hampton, NH, USA) and enhanced chemiluminescence (ECL) from Pierce (Rockford, IL, USA). The antibody against phospho-myosin-targeting subunit of myosin light chain phosphatase (phospho-MYPT1) at Thr855 $(1: 5,000)$ or MYPT1 was purchased from Upstate Biotechnology (Lake Placid, NY, USA) or BD Biosciences (San Jose, CA, USA) to check the level of RhoA/Rho-kinase activity (Wilson et al., 2005; Wooldridge et al., 2004). Anti-mouse IgM (goat) and anti- 


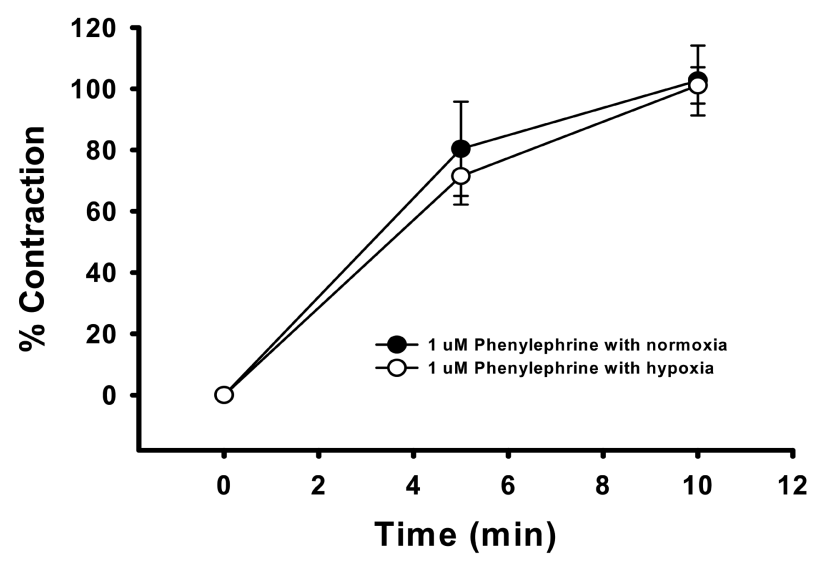

Fig. 1. Effect of pretreated no-aeration or aeration on phenylephrine-induced vascular contraction. Aeration or no aeration was applied $1 \mathrm{~min}$ before the addition of phenylephrine. Developed tension is expressed as a percentage of the maximum contraction to $50 \mathrm{mM} \mathrm{KCl}$. Data are expressed as means of 3-5 experiments with vertical bars showing SEM.

rabbit IgG (goat), conjugated with horseradish peroxidase, were used as secondary antibodies (1:2,000, 1:2,000, respectively, Upstate, Lake Placid, NY). Acetylcholine was dissolved in deionized water.

\section{Statistical analysis}

The data were expressed as mean \pm standard error of the mean (SEM). Student's unpaired $t$ test was used to determine the statistical significance of the means between two groups using SPSS 12.0 (SPSS Inc., Chicago, Illinois, U.S.A.). P values $<0.05$ were regarded as statistically significant.

\section{RESULTS}

\section{Effect of hypoxia on phenylephrine-induced con- traction of denuded aortae}

The absence of the endothelium was verified by the lack of relaxation after the addition of acetylcholine (1 $\mu \mathrm{M})$ to precontracted ring segments. Hypoxia didn't inhibit significantly phenylephrine-induced contraction regardless of endothelial function (Fig. 1) suggesting that MLCK or thin filament regulation might be of little importance.

\section{Effect of hypoxia on fluoride-induced contraction of denuded aortae}

Hypoxia completely inhibited Rho-kinase activator fluoride-induced contraction regardless of endothelial function (Fig. 2, Fig. 3) suggesting that Rho-kinase might be inhibited in the relaxation.

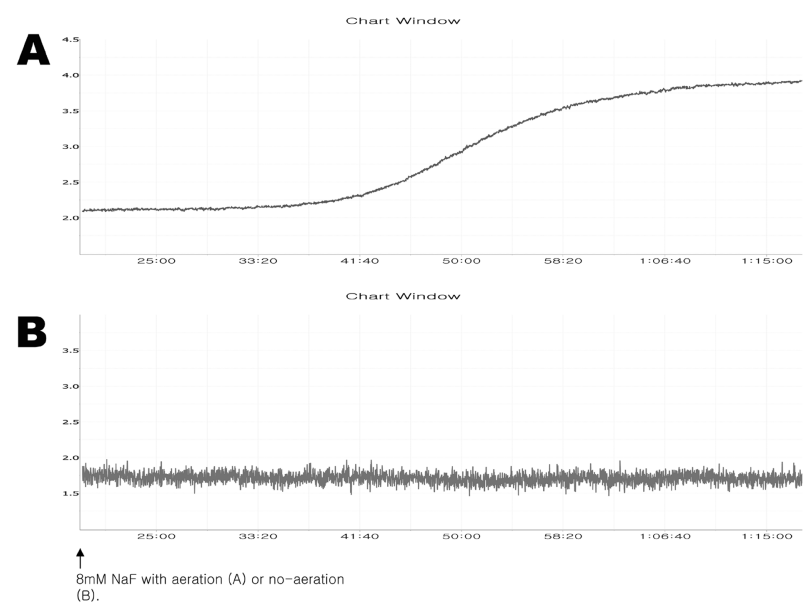

Fig. 2. A representative tracing of fluoride-induced vasoconstriction in the normoxia $(A)$ or hypoxia $(B)$ treated rat aorta.

\section{Effect of hypoxia on the level of phospho-MYPT1 at Thr-855}

To confirm the role of hypoxia in thick filament regulation of smooth muscle contractility, we measured the levels of myosin-targeting subunit of myosin light chain phosphatase (MYPT1) and phospho-MYPT1 in the muscles quick frozen after $30 \mathrm{~min}$ exposure to $8 \mathrm{mM}$ fluoride. $1 \mu \mathrm{M}$ Y-27632 was coadministrated with hypoxia. Interestingly, there was a significant decrease in the $8 \mathrm{mM}$ fluoride-induced MYPT1 phosphorylation at Thr855, the newly-highlighted site, instead of Thr697 (Wilson, et al.,

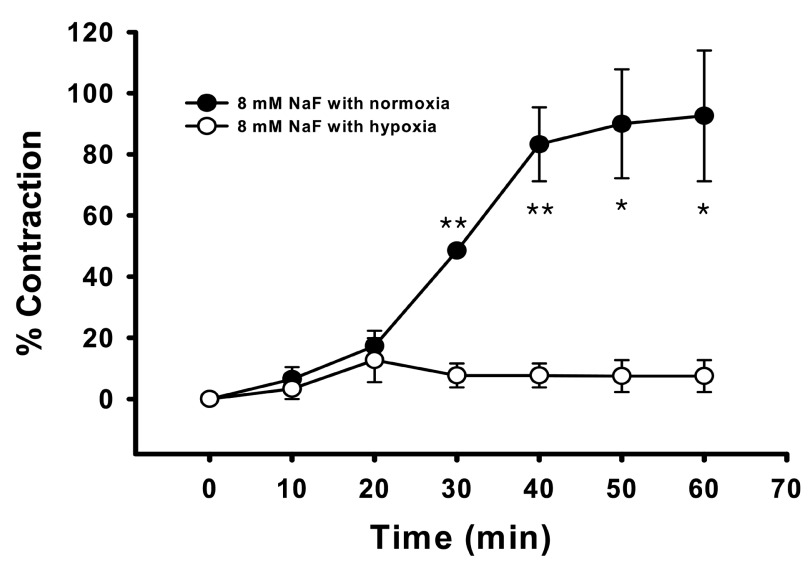

Fig. 3. Effect of pretreated no-aeration or aeration on fluorideinduced contraction. Aeration or no aeration was applied 1 min before the addition of fluoride. Developed tension is expressed as a percentage of the maximum contraction to 50 $\mathrm{mM} \mathrm{KCl}$. Data are expressed as means of 3-5 experiments with vertical bars showing SEM. ${ }^{\star} P<0.05$, ${ }^{\star \star} P<0.01$, presence versus absence of aeration. 
2005; Tsai and Jiang, 2006) in quick frozen hypoxiatreated rat aorta in the absence of endothelium compared to the vehicle treated rat aorta (Fig. 4). Thus thick or myosin filament regulation including myosin phosphatase activation through RhoA/Rho-kinase inactivation might be partly involved in decreased contractility in

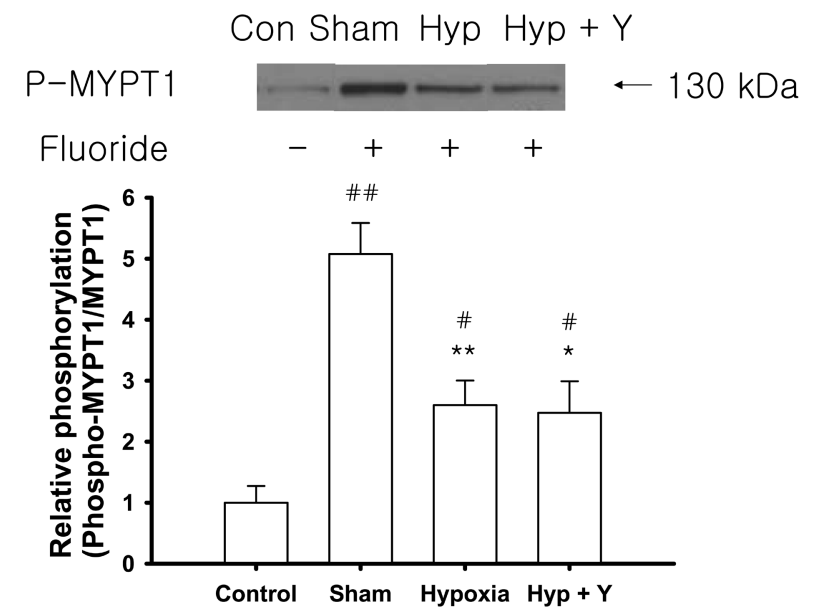

Fig. 4. Decreased phospho-MYPT1 $1_{\text {Thr855 }}$ protein levels in quick frozen hypoxia-added rat aorta in the absence of endothelium compared to the normoxia-added rat aorta contracted with $8 \mathrm{mM}$ fluoride. $1 \mu \mathrm{M}$ Y-27632 was coadministrated with hypoxia. Upper panel shows a typical blot and lower panel shows average densitometry results on the relative level of phospho-MYPT1/MYPT1. Data are expressed as means of 35 experiments with vertical bars showing SEM. ${ }^{*} P<0.05$, ${ }^{\star *} P<0.01$, \#\#P<0.01, versus sham or control respectively. Hyp: hypoxia; Y: $1 \mu \mathrm{M}$ Y-27632.

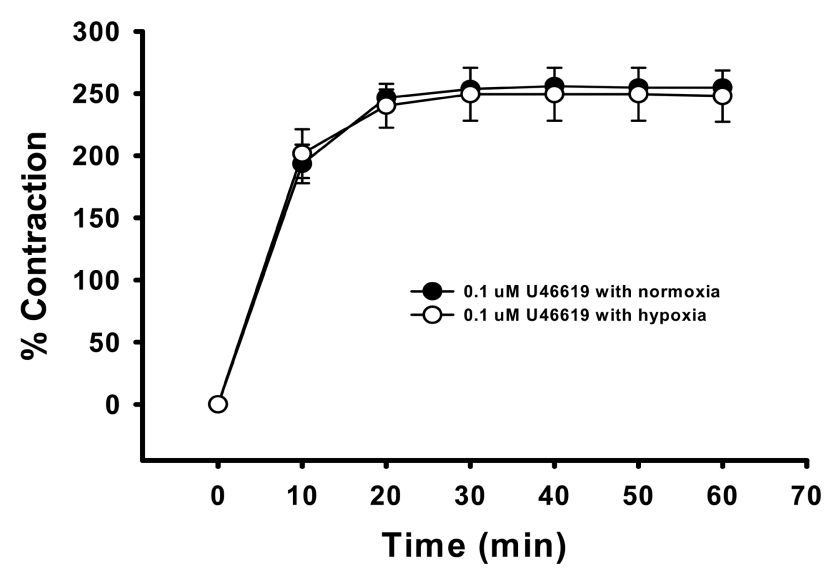

Fig. 5. Effect of pretreated no-aeration on U46619-induced contraction. Aeration or no aeration was applied $1 \mathrm{~min}$ before the addition of thromboxane $A_{2}$ mimetic. Developed tension is expressed as a percentage of the maximum contraction to 50 $\mathrm{mM} \mathrm{KCl}$. Data are expressed as means of 3-5 experiments with vertical bars showing SEM. hypoxia treated rat aorta.

\section{Effect of hypoxia on thromboxane $A_{2}$-induced con- traction of denuded aortae.}

Pretreated no-aeration or hypoxia didn't significantly inhibit Rho-kinase activator thromboxane $\mathrm{A}_{2}$ mimetic U46619-induced contraction regardless of endothelial function (Fig. 5) suggesting that Rho-kinase activity might be inhibited and other pathways than RhoA/Rho-kinase pathway might be involved in thromboxane $A_{2}$ mimetic $U$ 46619-induced $\mathrm{Ca}^{2+}$ sensitization such as $\mathrm{Ca}^{2+}$ entry or the phosphylation of extracellular signal-regulated kinase (ERK), protein kinase C-potentiated inhibitory protein for protein phosphatase type 1 (CPI-17) or integrin-linked kinase (ILK) $(47,48)$.

\section{DISCUSSION}

When the blood supply to a region is completely occluded leading to the hypoxia, arterioles in the region dilate because of myogenic relaxation, which occurs in response to the diminished stretch accompanying no blood flow, and changes in local chemical composition. Many of the same chemical changes occur in blooddeprived tissues that occur during metabolically induced active hyperemia. When a tissue's blood supply is blocked, $\mathrm{O}_{2}$ levels decrease in the deprived tissue because the tissue continues to consume $\mathrm{O}_{2}$, but no fresh supplies are being delivered. After the occlusion is removed, blood flow to the previously deprived tissue is transiently much higher than normal because the arterioles are widely dilated. Such a response is beneficial for rapidly restoring the local chemical composition including $\mathrm{O}_{2}$ or $\mathrm{CO}_{2}$ to normal. It was reported that the effect of hypoxia included vasodilation (Shimizu, et al., 2000). This study has investigated whether the inhibition of RhoA/ Rho-kinase activity contributes to the hypoxia-induced vascular relaxation in rat aortae contracted with a RhoA/ Rho-kinase activator such as fluoride or thromboxane $A_{2}$.

Two mechanisms are postulated for the development of contraction and myosin light chain $20 \mathrm{kDa}\left(\mathrm{MLC}_{20}\right)$ phosphorylation in smooth muscle. The first mechanism is that elevation in $\left[\mathrm{Ca}^{2+}\right]_{\mathrm{i}}$ activates a $\mathrm{Ca}^{2+}$-calmodulindependent myosin light chain kinase (MLCK), which in turn catalyzes phosphorylation of $\mathrm{MLC}_{20}$, leading to cross bridge cycling and contraction (Murphy, 1982). A decrease in the concentration of intracellular $\mathrm{Ca}^{2+}$ inactivates MLCK and allows MLC phosphatase to dephosphorylate $M L C_{20}$, resulting in muscle relaxation. A second mechanism of vascular smooth muscle contraction involves 
Rho-kinase (Amano, et al., 1996), whose activity is dependent on activation of the small GTP-binding protein RhoA. The RhoA/Rho-kinase pathway plays a key role in the $\mathrm{Ca}^{2+}$ sensitization of smooth muscle contraction, which is mainly induced by MLC phosphatase inhibition (Noda, et al., 1995; Somlyo and Somlyo, 2000).

The mechanism by which fluoride activates G-proteins has been clearly established (Kanaho, et al., 1985; Blackmore and Exton, 1986; Cockcroft and Taylor, 1987). It has been reported that the effect of fluoride on heterotrimeric $\mathrm{G}$ protein is the result of formation of $\mathrm{AlF}_{4}-$ from fluoride and trace amounts of aluminum, which can come from contamination of glassware (Zeng, et al., 1989; Chabre, 1990), and the structural similarity of $\mathrm{AlF}_{4}^{-}$to $\mathrm{PO}_{4}{ }^{3-}$ enabling to interact with GDP situated on the $\alpha$ subunit of the $G$ proteins where it can mimic GTP (Bigay, et al., 1985). Fluoride is also a classical Ser/Thr phosphatase inhibitor (Shenolikar and Nairn, 1991) and is routinely included in extraction buffers to prevent dephosphorylation of proteins on Ser and Thr residues by endogenous phosphatases. On the other hand, previous studies examining the mechanisms of thromboxane $A_{2}$ mimetic U46619-induced arterial contraction have reported variable findings with regard to the contraction related to Rho-kinase activation (Nobe and Paul, 2001; Tasaki, et al., 2003; Wilson, et al., 2005). Therefore, it was consistent with the possibility that the hypoxia could decrease fluoride- or thromboxane $\mathrm{A}_{2}$ mimetic-induced contraction inhibiting Rho-kinase activity.

The mechanisms by which Rho-kinase activation causes vascular contraction is an area of intense study, and several possibilities exist. For example, Rho-kinase phosphorylates myosin light chain phosphatase, resulting in decreased phosphatase activity and a buildup of phosphorylated myosin light chains (Somlyo and Somlyo, 2000; Pfitzer 2001). Rho-kinase has also been demonstrated to directly phosphorylate myosin light chains independently of myosin light chain kinase and phosphatase activity (Amano, et al., 1996). Recently, a role for Rho-kinase in vascular contraction evoked by sodium fluoride or thromboxane $A_{2}$ was established (Tsai and Jiang, 2006; Jeon, et al., 2006; Wilson, et al., 2005). Because fluoride-induced contraction was completely blocked by the hypoxia, it seems likely that under our experimental conditions, Rho-kinase inactivation may be the main pathway in the hypoxia-induced relaxation (Fig. 2, Fig. 3).

The main finding of the present study is that hypoxia completely inhibited fluoride-induced contraction regardless of endothelial function (Fig. 2, Fig. 3), and hypoxia didn't decrease thromboxane $A_{2}$-induced vasoconstriction regardless of endothelial function compared to fluoride-induced contraction (Fig. 5) suggesting some difference between the mechanisms of RhoA/Rho-kinase activators such as fluoride and thromboxane $A_{2}$. Therefore, we postulated that other pathways than RhoA/Rho-kinase pathway might be involved in thromboxane $A_{2}$ mimetic $U$ 46619-induced $\mathrm{Ca}^{2+}$ sensitization suggesting that hypoxia might not inhibit $\mathrm{Ca}^{2+}$ entry (Davis, et al., 2001; Low, 1996) or the phosphylation of extracellular signal-regulated kinase (ERK), protein kinase C-potentiated inhibitory protein for protein phosphatase type 1 (CPI-17) or integrin-linked kinase (ILK) (Deng, et al., 2001; Muranyi, et al., 2002). Furthermore, hypoxia decreased fluorideinduced phosphorylation of MYPT1 at Thr855, the newlyhighlighted site, instead of Thr697 (Fig. 4) suggesting inhibition of Rho-kinase activity and indicating that the relaxation mechanism of hypoxia is similar to that of $Y$ 27632. Therefore, suffice it to say that Rho-kinase inhibition is the major mechanism in hypoxia-induced vasorelaxation at least in the denuded and fluoride-contracted muscle, and the above-mentioned other pathways than RhoA/Rho-kinase pathway might be involved in thromboxane $\mathrm{A}_{2}$ mimetic-induced $\mathrm{Ca}^{2+}$ sensitization.

In summary, our results indicate that the hypoxia completely attenuated not thromboxane $\mathrm{A}_{2}$ - but fluorideinduced contraction in rat aortic rings regardless of endothelial function suggesting additional $\mathrm{Ca}^{2+}$ entry or the phosphorylation of ERK, CPI-17 or ILK besides RhoA/ Rho-kinase pathway existed in thromboxane $A_{2}$-induced contraction. The possible relaxing mechanism of the hypoxia in fluoride-induced contraction of the denuded muscle includes inhibition of Rho-kinase activity suggesting that other pathway is less important on the hypoxiainduced vasorelaxation in agonist-dependent contraction of the denuded muscle. Subsequent interactions between myosin phosphatase and weakened Rho-kinase may lead to attenuated $\mathrm{Ca}^{2+}$ sensitivity of the myofilament and vascular relaxation.

\section{REFERENCES}

Aalkjaer, C. and Lombard, J. H. (1995). Effect of hypoxia on force, intracellular $\mathrm{pH}$ and $\mathrm{Ca}^{2+}$ concentration in rat cerebral and mesenteric small arteries. J. Physiol. 482, 409-419.

Amano, M., Ito, M., Kimura, K., Fukata, Y., Chihara, K., Nakano, T., Matsuura, Y. and Kaibuchi, K. (1996). Phosphorylation and activation of myosin by Rho-associated kinase (Rhokinase). J. Biol. Chem. 271, 20246-20249.

Bigay, J., Deterre, P., Pfister, C. and Chabre, M. (1985). Fluoroaluminates activate transducin-GDP by mimicking the gamma-phosphate of GTP in its binding site. FEBS Lett. 191, 
181-185

Blackmore, P. F. and Exton, J. H. (1986). Studies on the hepatic calcium-mobilizing activity of aluminum fluoride and glucagon. Modulation by CAMP and phorbol myristate acetate. $J$. Biol. Chem. 261, 11056-11063.

Chabre, M. (1990). Aluminofluoride and beryllofluoride complexes: a new phosphate analogs in enzymology. Trends. Biochem. Sci. 15, 6-10.

Cockcroft, S. and Taylor, J. A. (1987). Fluoroaluminates mimic guanosine 5'-[gamma-thio]triphosphate in activating the polyphosphoinositide phosphodiesterase of hepatocyte membranes. Role for the guanine nucleotide regulatory protein $\mathrm{Gp}$ in signal transduction. Biochem. J. 241, 409-414.

Davis, M. J., Wu, X., Nurkiewicz, T. R., Kawasaki, J., Gui, P., Hill, M. A. and Wilson, E. (2001). Regulation of ion channels by protein tyrosine phosphorylation. Am. J. Physiol. 281, $\mathrm{H} 1835-\mathrm{H} 1862$.

Deng, J. T., Van Lierop, J. E., Sutherland, C. and Walsh, M. P. (2001). Ca ${ }^{2+}$-independent smooth muscle contraction: a novel function for integrin-linked kinase. J. Biol. Chem. 276, 1636516373.

Ishida, Y. and Honda, H. (1992). Underlying mechanisms for hypoxia-induced relaxation of the guinea-pig isolated aorta. Jpn. J. Pharmacol. 58 (Suppl. 2), 307.

Jeon, S. B., Jin, F., Kim, J. I., Kim, S. H., Suk, K., Chae, S. C., Jun, J. E., Park, W. H. and Kim, I. K. (2006). A role for Rho kinase in vascular contraction evoked by sodium fluoride. Biochem. Biophys. Res. Commun. 343(1), 27-33.

Kitazawa, T., Masuo, M. and Somlyo, A. P. (1991). Proteinmediated inhibition of myosin light-chain phosphatase in vascular smooth muscle. Proc. Natl. Acad. Sci. USA 88, 93079310.

Kanaho, Y., Moss, J. and Vaughan, M. (1985). Mechanism of inhibition of transducin GTPase activity by fluoride and aluminum. J. Biol. Chem. 260, 11493-11497.

Tsai, M. H. and Jiang, M. J. (2006). Rho-kinase-mediated regulation of receptor-agonist-stimulated smooth muscle contraction. Pflugers Arch. 453, 223-232.

Low, A. M. (1996). Role of tyrosine kinase on $\mathrm{Ca}^{2+}$ entry and refilling of agonist-sensitive $\mathrm{Ca}^{2+}$ stores in vascular smooth muscles. Can. J. Physiol. Pharmacol. 74, 298-304.

Muranyi, A., MacDonald, J. A., Deng, J. T., Wilson, D. P., Haystead, T. A., Wlash, M. P., Erdodi, F., Kiss, E., Wu, Y. and Hartshorne, D. J. (2002). Phosphorylation of the myosin phosphatase target subunit by integrin-linked kinase. Biochem. J. 366, 211-216.

Murphy, R. A. (1982). Myosin phosphorylation and crossbridge regulation in arterial smooth muscle. Hypertension 4, 3-7.

Nobe, K. and Paul, R. J. (2001). Distinct pathways of $\mathrm{Ca}^{2+}$ sensitization in porcine coronary artery: effects of Rho-related kinase and protein kinase $\mathrm{C}$ inhibition on force and intracellular $\mathrm{Ca}^{2+}$. Circ. Res. 88(12), 1283-1290.

Noda, M., Yasuda-Fukazawa, C., Moriishi, K., Kato, T., Okuda, T., Kurokawa, K. and Takuwa, Y. (1995). Involvement of rho in GTP gamma S-induced enhancement of phosphorylation of $20 \mathrm{kDa}$ myosin light chain in vascular smooth muscle cells: inhibition of phosphatase activity. FEBS Lett. 367, 246-250.

Pfitzer G. Invited reviews: regulation of myosin light chain phosphorylation in smooth muscle. J Appl Physiol 2001; 91:497503.

Shimizu, S., Bowman, P. S., Thorne, G. III and Paul, R. J. (2000). Effects of hypoxia on isomeric force, intracellular $\mathrm{Ca}^{2+}, \mathrm{pH}$, and energetics in porcine coronary artery. Circ. Res. 86, 862-870.

Sakurada, S., Takuwa, N., Sugimoto, N., Wang, Y., Seto, M., Sasaki, Y. and Takuwa, Y. (2003). $\mathrm{Ca}^{2+}$-dependent activation of Rho and Rho-kinase in membrane depolarizationinduced and receptor stimulation-induced vascular smooth muscle contraction. Circ. Res. 93, 548-556.

Shenolikar, S. and Nairn, A. C. (1991). Protein phosphatases: recent progress. Adv. Second. Messenger. Phosphoprotein. Res. 23, 1-121.

Somlyo, A. P. and Somlyo, A. V. (1994). Signal transduction and regulation in smooth muscle. Nature 372, 231-236.

Somlyo, A. P. and Somlyo, A. V. (1998). From pharmacomechanical coupling to G- proteins and myosin phosphatase. Acta. Physiol. Scand. 164, 437-448.

Somlyo, A. P. and Somlyo, A. V. (2000). Signal transduction by G-proteins, rho-kinase and protein phosphatase to smooth muscle and non-muscle myosin II. J. Physiol. 522, 177-185.

Tasaki, K., Hori, M., Ozaki, H., Karaki, H. and Wakabayashi, I. (2003). Difference in signal transduction mechanisms involved in 5-hydroxytryptamine- and U46619-induced vasoconstrictions. J. Smooth Muscle Res. 39, 107-117.

Uehata, M., Ishizaki, T., Satoh, H., Ono, T., Kawahara, T., Morishita, T., Tamakawa, H., Yamagami, K., Inui, J., Maekawa, M. and Narumiya, S. (1997). Calcium sensitization of smooth muscle mediated by a Rho-associated protein kinase in hypertension. Nature 389, 990-994.

Wier W. G. and Morgan, K. G. (2003) $\alpha 1$-Adrenergic signaling mechanisms in contraction of resistance arteries. Rev Physiol Biochem Pharmacol 150, 91-139.

Wilson, D. P., Susnjar, M., Kiss, E., Sutherland, C. and Walsh, M. P. (2005). Thromboxane $A_{2}$-induced contraction of rat caudal arterial smooth muscle involves activation of $\mathrm{Ca}^{2+}$ entry and $\mathrm{Ca}^{2+}$ sensitization: Rho-associated kinase-mediated phosphorylation of MYPT1 at Thr-855, but not Thr-697. Biochem. J. 389, 763-774.

Wooldridge, A. A., MacDonald, J. A., Erdodi, F., Ma, C., Borman, M. A., Hartshorne, D. J., and Haystead, T. A. (2004). Smooth muscle phosphatase is regulated in vivo by exclusion of phosphorylation of threonine 696 of MYPT1 by phosphorylation of Serine 695 in response to cyclic nucleotides. $J$. Biol. Chem. 279, 34496-34504.

Young, M. A. and Vatner, S. F. (1986). Regulation of large coronary arteries. Circ. Res. 59, 579-596.

Zeng, Y. Y., Benishin, C. G. and Pang, P. K. (1989). Guanine nucleotide binding proteins may modulate gating of calcium channels in vascular smooth muscle. I. Studies with fluoride. J. Pharmacol. Exp. Ther. 250, 343-351. 